\title{
Le diagnostic anténatal des génodermatoses
}

Le diagnostic prénatal des maladies génétiques de la peau repose encore le plus souvent sur la biopsie cutanée par fotoscopie, suivie d'un examen structural. Cependant, la reconnaissance des mécanismes de ces maladies permet aujourd'hui d'adjoindre à cette étude des analyses immunologiques et enzymatiques et, surtout, de la remplacer par un diagnostic précoce reconnaissant la lésion de l'ADN sur des biopsies de villosités choriales pratiquées à la $10^{\mathrm{e}}$ semaine de gestation.

\section{Claudine Blanchet-Bardon}

\section{ADRESSE}

Cl. Blanchet-Bardon: médecin des hôpitaux. Clinique des maladies cutanćes ( $\mathrm{Pr}$ Louis Dubertret), hôpital Saint-Louis, 1, avenue

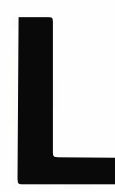

es génodermatoses regroupent environ 200 maladies génétiques à expression cutanée. Alors qu'il y a une dizaine d'années, seul un conseil génétique était proposé aux couples à risques, la gravité de certaines maladies cutanées, ou leur caractère létal, ainsi que l'absence de thérapeutique efficace ont justifié le recours à un diagnostic anténatal. Pendant près de dix ans, ce diagnostic anténatal a été fait par abord direct du fœtus, c'est-à-dire par fœtoscopie et biopsie de peau, à un terme tardif de 20 semaines de gestation. Plus récemment, les techniques de génétique moléculaire ont permis d'améliorer les performances de ce diagnostic et font penser que dans la prochaine décennie le diagnostic anténatal en dermatologie pourra connaître un terme plus précoce : celui de la biopsie de trophoblaste à 10 semaines.

\section{Diagnostics anténatals par fotoscopie et microscopie électronique}

Introduites par 1972 par Valenti [1], ce n'est qu'en 1980 que les premiè- res biopsies cutanées fœtales ont été réalisées et que la fœtoscopie a été appliquée au diagnostic anténatal des génodermatoses sévères. Cette technique endoscopique permet une visualisation directe du fœtus et du placenta, et permet d'obtenir des échantillons de peau fotale pour une étude ultrastructurale, une culture de tissu ou une analyse biochimique. Grâce à une meilleure connaissance de la technique, de ses risques et du développement de la peau fœtale, cette méthode a connu un essor important et un élargissement de ses indications à de nombreuses autres maladies héréditaires.

Rappel sur la structure de la peau au moment de la fotoscopie, c'està-dire entre 20 et 21 semaines de gestation [2, 3]

A cette période, l'épiderme est encore recouvert par le périderme. L'examen ultrastructural montre qu'il est constitué par des cellules légèrement aplaties à leur base présentant une ou deux protusions globulaires se projetant dans l'activité amniotique. La surface libre de ces cellules épidermiques, en contact avec le liquide amniotique, présente de nombreuses 
villosités. Ces cellules sont attachées entre elles et aux cellules de la couche intermédiaire grâce à des desmosomes. $\mathrm{La}$ fonction principale du périderme est ainsi de permettre les échanges entre le fœtus et le liquide amniotique.

Les couches plus profondes de l'épiderme sont constituées

- d'une couche de cellules basales, cubiques, apparaissant claires en ultrastructure du fait de leur pauvreté en glycogène,

- de trois couches de cellules intermédiaires riches en glycogène et en filaments de kératine.

C'est à partir de la couche intermédiaire que se développeront les couches épineuse, granuleuse et cornée. Cette période est caractérisée par la présence de cellules granuleuses contenant des grains de kératohyaline, observées uniquement au niveau des œstium folliculaires alors que la kératinisation n'a pas encore commencé au niveau de l'épiderme interfolliculaire.

L'apparition des follicules pilaires et des cheveux se fait selon une direction cranio-caudale. Les follicules pileux sont bien développés à vingt semaines. Cependant, les infundibulum pilaires traversent parfois l'épiderme de façon presque horizontale, donnant ainsi sur les coupes un aspect anormal de longues stries d'épiderme kératinisé qu'il ne faut pas confondre avec un début de kératinisation interfolliculaire.

Au terme de la fœtoscopie, la zone de jonction dermo-épidermique est complètement formée. Tous les composants de la zone de jonction dermoépidermique sont présents : les hémidesmosomes avec leur plaque dense sous-hémidesmosomiale dans la lamina lucida, les fibrilles d'ancrage, les fibres d'ancrage et les microfibrilles encore appelés fibres oxytalanes.

Enfin, les techniques d'immunofluorescence permettent de mettre en évidence à cet âge les différents antigènes de la zone de jonction dermoépidermique. L'antigène de la pemphigoïde bulleuse apparaît dès le troisième mois de la vie intra-utérine, ainsi que la laminine, le collagène de type IV et celui de type VII, la fibronectine.

Les mélanocytes sont présents au niveau de l'épiderme interfolliculaire $m / s n^{\circ} 4$ vol. 9, avril 93 mais ne comportent le plus souvent que des mélanosomes de stades II et III. Au niveau du bulbe pilaire, en revanche, les mélanocytes sont mûrs et comportent de nombreux mélanonomes de stade IV. Le derme étant encore très immature, il n'est pas possible de distinguer le derme papillaire du derme réticulaire. Quant aux fibres élastiques présentes à ce terme, il est pratiquement impossible de les reconnaître histologiquement par leur structure et leur organisation.

Les glandes sébacées sont fonctionnelles dès la $13^{e}-15^{e}$ semaine de gestation et participent chez le fotus à la formation du vernix caseosa.

Ce n'est que plus tardivement, à la fin du $5^{\mathrm{e}}$ mois de gestation, que les glandes eccrines se développent respectivement aux régions palmoplantaires et aux régions axillaires.

\section{Fatoscopie}

La fœtoscopie pour diagnostic anténatal par biopsie cutanée fœtale est réalisée entre la $19^{e}$ et la $22^{e}$ semaine de gestation. Elle est obligatoirement précédée d'une échographie qui précisera l'âge gestationnel, la viabilité fœtale, le nombre de fœtus, la localisation du placenta, la position du fœtus. Par ailleurs, cette fœtoscopie est faite sous contrôle échographique continu et en temps réel, ce qui permet une meilleure localisation et facilite le prélèvement. Le fœtoscope utilisé est un endoscope rigide comportant un éclairage par fibre optique transmettant la lumière à la cavité amniotique. Le fœtoscope est introduit à l'intérieur de la cavité amniotique à l'aide d'un trocart et d'une canule. La canule possède une entrée en Y permettant l'introduction d'une aiguille très fine pour l'utilisation d'une pince à biopsie qui permet d'obtenir des prélèvements de $1 \mathrm{~mm}^{3}$. Le siège des biopsies est déterminé le plus souvent suivant la maladie à rechercher. Il est pratiqué en moyenne trois prélèvements par fotus et les biopsies sont observées à la loupe binoculaire, permettant de préciser si les prélèvements sont bien de la peau [4].

Principales indications des diagnostics anténatals par fotoscopie et microscopie électronique

- Diagnostic anténatal des épidermolyses bulleuses héréditaires

Comme le montre le Tableau I, toutes les épidermolyses bulleuses décrites ont des marqueurs suffisants pour assurer un diagnostic anténatal fiable. Cependant, les indications doivent être discutées, d'une part, avec la famille et, d'autre part, selon les règles d'éthique. Les deux diagnostics anténatals demandés le plus communément dans notre série sont celui de l'épidermolyse bulleuse jonctionnelle létale d'Herlitz et celui de l'épidermolyse bulleuse récessive dystrophique d'Hallopeau-Siemens [5-7].

- Épidermolyse bulleuse létale jonctionnelle d'Herlitz (figure 1)

C'est la plus grande indication du fait de la létalité de la maladie avec un espoir de vie n'excédant pas une année. Le marqueur ultrastructural pour le diagnostic anténatal est l'absence ou la dysplasie des hémidesmosomes au niveau de la zone de jonction dermo-épidermique. Cela aboutit à un décollement au niveau de la pars lucida qui peut être visible sur les biopsies de peau après fœtoscopie. L'anticorps monoclonal GB3 qui reconnaît la nicéine n'est pas utilisé en routine pour le diagnostic anténatal car l'immunofluorescence avec le GB3 peut être positive dans certaines épidermolyses bulleuses jonctionnelles comme les non-létales et celles associées à une sténose du pylore. La plupart des familles françaises n'ayant pas encore été étudiées par le GB3, il nous a semblé plus sûr de nous tenir à l'ultrastructure dont le marqueur vaut pour toutes les hypoplasies des hémidesmasomes [8].

- Épidermolyses bulleuses récessives dystrophiques type Hallopeau-Siemens (figure 2)

Ces types d'épidermolyse bulleuse représentent un large spectre clinique où se retrouvent un certain nombre de dénominateurs communs dès la première enfance : synéchie des extrémités, atteinte muqueuse, sténose de l'œsophage, retard staturo-pondéral, anémie inflammatoire. Le décès peut survenir à tout moment par septicémie et, secondairement, dès la deuxième décennie, par dénutrition, altération de l'état général ou épithélioma spinocellulaire sur plaies ou 
hyperkératoses chroniques. Le marqueur du diagnostic postnatal et prénatal de ce type d'EB est l'absence ou la dystrophie des fibres d'ancrage constituécs de collagène VII. L'EB dystrophique d'Hallopeau-Siemens a été récemment localisée sur le chromosome III au niveau du gène du collagène VII [9].

\section{- Quel est l'avenir?}

Les travaux sur la BM600 ou nicéine vont rapidement aboutir à un diagnostic anténatal plus précoce (voir l'article de G. Meneguzzi et al., p. 387 de ce numéro). La localisation de l'épidermolyse bulleuse dystrophique récessive d'Hallopeau-Siemens sur le chromosome III et la recherche des mutations permettent dès maintenant un diagnostic précoce à 10 semaincs par biopsie de trophoblaste. La double localisation de l'épidermolyse bulleusc herpétiforme de Dowling-Meara sur les chromosomes 12 à 17 laissc espérer également un diagnostic précoce dès que les familles à risque auront pu être étudiées [10-11].

\section{- Troubles de la kératinisation}

Les troubles de la kératinisation sont définis par une desquamation permanente de la peau associée ou non à un érythème et à d'autres anomalies générales.

\section{- Fætus Arlequin}

Le fœtus Arlequin a été le premier diagnostic anténatal décrit parmi les troubles de la kératinisation [12]. Le

\section{Tableau I}

\section{CLASSIFICATION DES ÉPIDERMOLYSES BULLEUSES CONGÉNITALES}

Ha, Odland GF. Regional development in the human epidermis in the first trimester embryo and the sccond trimester fetus (ages related to the timing of the amniocentesis and fetal biopsy). $J$ Invest Dermatol 1980 ; 74 : 161-8.

4. Rodeck CH. Fetoscopy guided by real time ultrasound for purc fetal blood samples, fetal skin samples and examination of fctus in utero. Br J Obstet Gynaecol $1980 ; 87$ : 449-56.

5. Blanchet-Bardon $\mathrm{Cl}$, Dumcz $\mathrm{Y}, \mathrm{Naz}-$ zaro V, Mimoz C, Puissant A. Le diagnostic anténatal des épidermolyses bullcuses héréditaires. Ann Dermatol Venereol 1987 ; 114 : 525-39.

6. Anton-Lamprecht I. Prenatal diagnosis of genetic disorders of the skin by means of electron microscopy. Hum Genet 1981 ; 59 : 392-405.

7. Fine JD, Bauer EA, Briggaman RA, Carter DM, Eady RAJ, Esterly NB, Holbrook KA, Hurwitz S, Johnson I, Lin A, Pcarson R, Sybert VP. Revised clinical and laboratory criteria of subtypes of inherited epidermolysis bullosa. J Am Acad Dermatol $1991 ; 24: 119-35$

8. Verrando $\mathrm{P}$, Blanchct-Bardon $\mathrm{Cl}$, Pisani A, Thomas L, Cambazard F, Eady RAJ, Schoficld O, Ortonne J-P. Monoclonal antibody GB3 defines a widespread defect of several basement membranes and a keratinocyte dysfunction in patients with lethal junctional cpidermolysis bullosa. Lab Invest $1991 ; 64,1: 85-92$.

9. Hovnanian A, Duquesnoy P, BlanchetBardon $\mathrm{Cl}$, Knowlton RG, Amsellem S, Lathrop M, Dubertret L, Uitto J, Gossens M. Genetic linkage of recessive dystrophic epidermolysis bullosa to the VII colla-

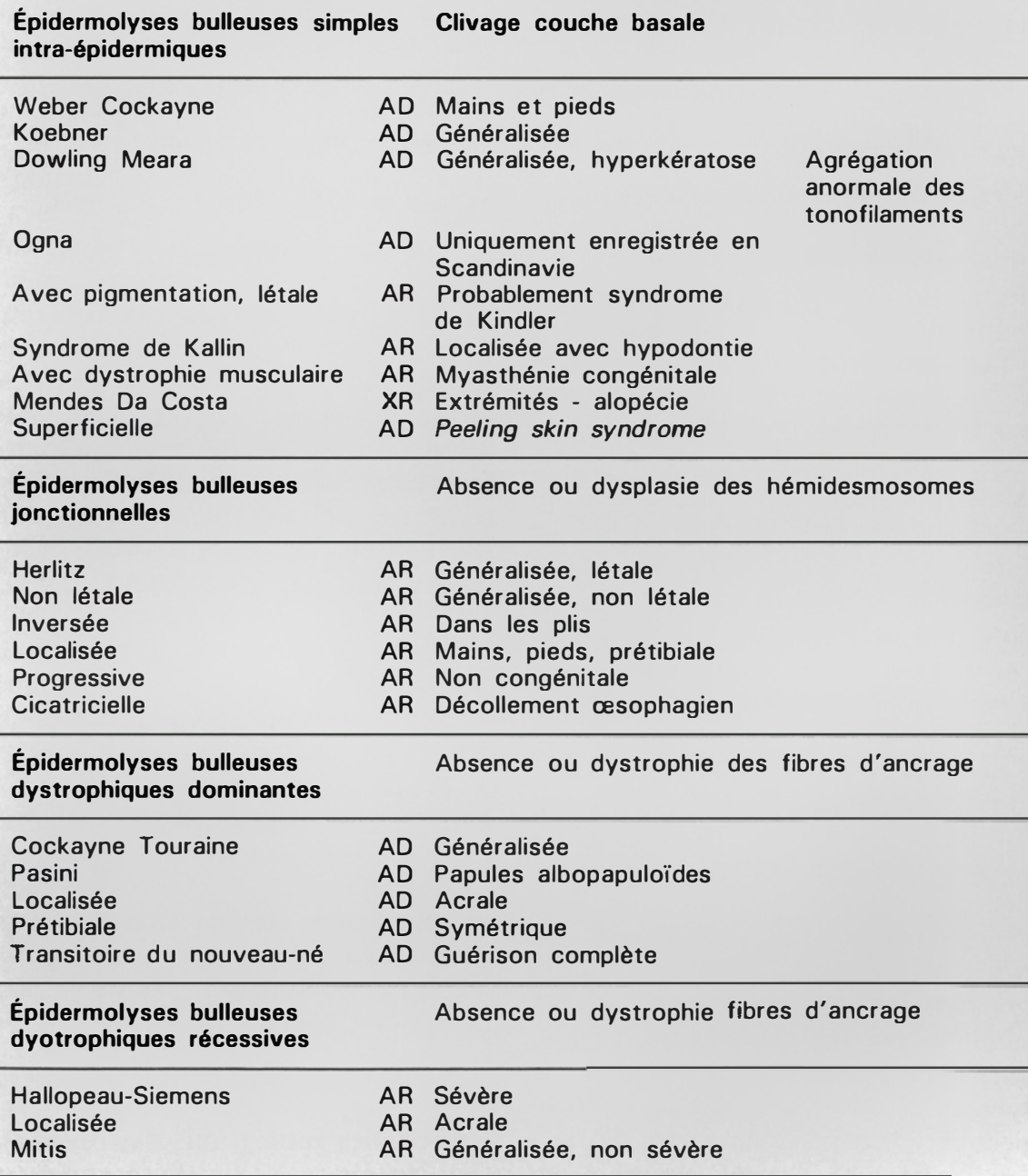

$A R$ : autosomique récessif. $A D$ : autosomique dominant. $X R$ : récessif lié à $I ' X$. 

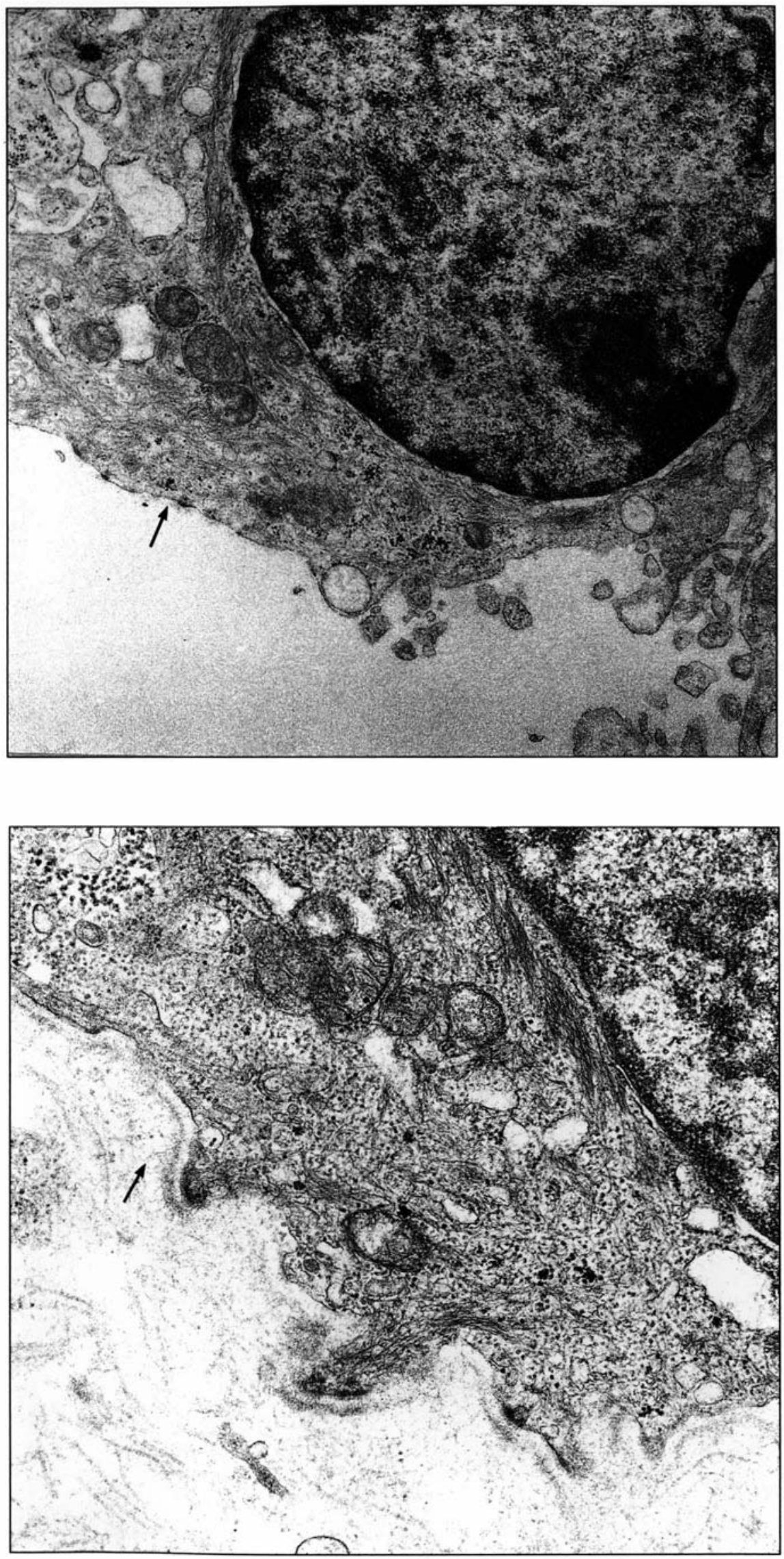

$\mathrm{m} / \mathrm{s} n^{\circ} 4$ vol. 9, avril 93
Figure 1. Épidermolyse bulleuse létale d'Herlitz. Peau décollée lors du prélèvement. Noter les hémidesmosomes dysplasiques. (x 30 000.)

Figure 2. Épidermolyse bulleuse récessive dystrophique. Peau non décollée. Noter l'absence de fibres d'ancrage. (x 20 000.) 


\section{RÉFÉRENCES}

10. Lanc EB, Rugg EL, Navsaria $H$, Leigh IM, Heagerty A HM, IshidaYamamoto A, Eady RAJ. A mutation in the conscrved helix termination peptide of keratin 5 in hercditary skin blistcring. Nature $1992 ; 356: 244-6$.

11. Coulombc PA, Hutton ME, Letai A, Hebert A, Paller AS, Fuchs E. Point mutations in human keratin 14 genes of epidermolysis bullosa simplex patients : genetic and functionnal analyses. Cell 1991; 66 : 1301-11.

12. Arnold ML, Anton-Lamprecht I. Problems in prenatal diagnosis of the ichtyosis congenital group. Hum Genet 1985; 71 : 301-11.

13. Arnold ML, Anton-Lamprecht I. Prenatal diagnosis of epidermal disorders. Curr Probl Dermatol 1987 ; 16 : 120-8.

14. Bakharev VA, Aivazyan AA, Karetnikova NA, Mordovtsev VN, Yantovsky YuR. Fetal skin biopsy in prenatal diagnosis of some genodcrmatosis. Prenat Diagn $1990 ; 10: 1-12$.

15. Holbrook EB, Willcy AM. Abortions because of unavailability of prenatal diagnosis. Lancet 1981 ; 2 : 936.

16. Holbrook KA, Dale BA, Sybert VP, Sagebiel RW. Epidermolytic hyperkeratosis : Ultrastructure and biochemistry of skin and amniotic fluid cells from two affected fatuses and a newborn infant. $J$ Invest Dermatol $1983 ; 80: 222-7$.

17. Cheng J, Syder AJ, Yu QC, Letai A, Paller AS, Fuchs E. The genetic basis of epidermolytic hyperkeratosis : a disorder of differentiation-specific epidermal keratin genes. Cell 1992; 70 : 811-9. fœtus Arlequin est une ichtyose grave, marquée dès la naissance par une hyperkératinisation massive quadrillée de fissures profondes, d'où le nom de fœtus Arlequin. Il existe également un ectropion* et un éclabion* Le fœtus Arlequin est incompatible avec la vie. Le marqueur ultrastructural le plus fiable est la présence d'un épaississement anormalement précoce des membranes cytoplasmiques des couches superficielles de l'épiderme et la présence de vésicules cerclées d'une membrane épaisse. Mais, cette hyperkératose précoce et importante, qui a été parfois observée à 22 semaines, ne peut être retrouvée qu'à 24 semaines du fait de l'hétérogénéité de cette maladie.

- Bébé collodion et ichtyoses congénitales Le bébé collodion est un aspect clinique à la naissance : le nouveau-né est entouré d'une membrane collodionnée traversée par les cheveux et éversant tous les orifices. Cet état clinique à la naissance ne préjuge pas de l'évolution ultérieure : ichtyose lamellaire, érythrodermie congénitale ichtyosiforme sèche, etc.

Au début du diagnostic anténatal par fœtoscopie et microscopie électronique, nous pensions qu'à l'instar du fœtus Arlequin où avait été décrite une kératinisation très précoce, le syndrome du bébé collodion (hyperkératinisation présente dès la naissance) pourrait être diagnostiqué par la constatation d'une hyperkératinisation entre 20 et 22 semaines de gestation. L'expérience nous a montré assez rapidement que ce marqueur ultrastructural n'était pas suffisant pour établir un diagnostic fiable du syndrome du bébé collodion. Donc, depuis quelques années, un certain nombre de difficultés ont été rapportées dans l'élaboration du diagnostic anténatal des ichtyoses congénitales [13]. Ces difficultés sont dues essentiellement au développement tardif de la kératinisation chez le fœutus normal, vers la $24^{\mathrm{e}}$ semaine de gestation. Ainsi, certains auteurs [14] ont axé leur diagnostic anténatal sur la recherche d'anomalies morphologiques au niveau des follicules pilaires où la kératinisation est plus précoce :

\footnotetext{
* Voir glossaire, ci-contre.
}

\section{GLOSSAIRE}

Ectropion : éversement des paupières surtout inférieures.

Eclabion : éversement des lèvres.

Maladie de Fabry : récessive liée à l'X. Déficit en $\gamma$-galactosidase A. Touche principalement la peau (angiokératome), le rein (insuffisance rénale), le cerveau (hémorragie cérébrale).

Anémie de Fanconi: Autosomique récessive. Syndrome d'instabilité chromosomique. Pancytopénie, leucémie, hyperpigmentation, hypoplasie génitale et microcéphalie.

Syndrome de Farber ou Lipogranulomatose : autosomique récessif. Déficit en céramidase. In filtration généralisée des organes dont la peau par un granulome histiocytome.

Syndrome de Menkes : récessif lié à l'X, Kinky hair (cheveux crépus), peau épaisse et sèche. Profonde détérioration neurologique progressive avec hypertonie, épilepsie... Diagnostic anténatal par démonstration d'incorporation augmentée du cuivre dans les cellules amniotiques cultivées.

Mucolipidose de type II ou syndrome de Leroy I. Cell : autosomique récessif. Déficit en $N$-acétyl glucosamine 1 phosphotransférase. Hypertrophie gingivale, limitation articulaire, peau épaisse, scléreuse.

Maladie de Wolman ou Xanthomatose familiale primitive : autosomique récessive. Déficit en lipase acide lysosomiale. Infiltration généralisée des organes y compris la peau par des cellules spumeuses contenant des triglycérides et du cholestérol.

Syndrome d'Ehler-Danlos de type VI : ausotomique récessif. Déficit en lysyl hydroxylase. Laxité ligamentaire, peau hyperextensible, anomalies oculai res. Kératocome.

Fucosidose : autosomique récessif. Déficit en $\alpha$-fucosidase. Retard mental et moteur sévère. Multiples télangiectasies.

Maladie de Gunther ou porphyrie érythropoïétique congénitale : autosomique récessif. Déficit en uroporphyrinogène III synthétase. Urines rouges. Photosensibilité. Cicatrices.

Maladie de Refsum : autosomique récessif. Déficit en oxydase de l'acide phytanique. Polynévrite. Rétinite pigmentaire, signes cérébelleux, ichtyose. 
20-21 semaines de gestation. Cependant, là encore, il ne s'agit pas d'un marqueur suffisamment spécifique puisqu'il est rapporté la naissance d'un enfant atteint d'érythrodermie congénitale ichtyosiforme sèche chez lequel aucune anomalie de structure des follicules pilaires ou de l'épithélium interfolliculaire n'a été détectée à 20 semaines de gestation. Plusieurs facteurs interviennent probablement dans les faux diagnostics : la mauvaise localisation du site biopsique, l'hétérogénéité des maladies, des expressions cliniques et des variations individuelles dans le moment du début de la kératinisation [15]. La localisation du site biopsique doit tenir compte du gradient craniocaudal de différenciation, mais, en plus, il semble que la kératinisation de l'épiderme interfolliculaire commence par petites zones sur toute la surface du corps. Ainsi, il est possible d'obtenir chez le même fœtus des biopsies où il existe une kératinisation continue ou d'autres où la kératinisation n'a pas encore commencé [14]. Cela incite donc à être extrêmement prudent en matière de diagnostic anténatal des troubles de la kératinisation et en particulier du syndrome du bébé collodion.

\section{- Érythrodermie congénitale ichtyosiforme} bulleuse

L'érythrodermie congénitale ichtyosiforme bulleuse est marquée dès la naissance par un aspect de bébé ébouillanté, érythémateux avec décollements cutanés. Ce n'est que dans la deuxième enfance qu'une importante hyperkératose s'installe, toujours associée à une fragilité cutanée. Le marqueur ultrastructural permettant le diagnostic est la cytolyse des kératinocytes par rupture du complexe fonctionnel desmosomes-tonofilaments et l'agrégation anormale des tonofilaments en mottes cytoplasmiques et en écorces périnucléaires. Entre 20 et 22 semaines de gestation, ce diagnostic anténatal par biopsie cutanée du fœtus peut être délicat car l'agrégation des tonofilaments ne peut pas encore s'être produite et la cytolyse peut ressembler à un artefact de fixation, en particulier à la dissolution du glycogène dans les solvants utilisés [16].

L'érythrodermie congénitale ichtyosi$\mathrm{m} / \mathrm{s} \mathrm{n}^{\circ} 4$ vol. 9, avril 93 forme bulleuse a été récemment localisée par deux équipes différentes au niveau du gène de la kératine 1 et de la kératine 10 [17-18].

\section{Trichothiodystrophie}

Maladie autosomique récessive caractérisée par des cheveux cassants avec un contenu en protéines soufrées réduit, un retard mental et staturopondéral, la trichothiodystrophie peut être associée à une ichtyose congénitale. Dans le cas où l'étude de la réparation de l'ADN par trophoblaste n'est pas probante, un diagnostic anténatal peut être établi par biopsie $\mathrm{du}$ cuir chevelu et polarisation du cheveu fotal montrant l'aspect en "queue de tigre "spécifique [19].

- Dysplasie ectodermique anhydrotique de Christ-Siemens-Touraine

Cette affection est une génodermatose liée à l'X. Le diagnostic anténatal ne peut donc intéresser que les garçons. Le diagnostic anténatal peut se gérer de deux façons : (1) par foetoscopie et biopsie de peau du feetus à 20 semaines avec étude du follicule pilo-sébacé qui est absent dans cette maladie, les glandes sudorales ne pouvant pas être prises en considération car non développées au terme de la fœtoscopie. Ce diagnostic tardif n'est réservé qu'aux familles non informatives ou qui n'ont pu être étudiées en génétique moléculaire ; (2) en génétique moléculaire par biopsie de trophoblaste chez les familles préétudiées et informatives.

\section{- Troubles pigmentaires}

Leur diagnostic n'est accessible que par l'intermédiaire du mélanocyte du bulbe pilaire. On utilise donc des biopsies du cuir chevelu étant donné l'abondance des bulbes pilaires.

Nous pouvons ainsi diagnostiquer in utero l'albinisme tyrosinase négatif et surtout des maladies immunohématologiques associant un albinisme partiel et un déficit de l'immunité telles que la maladie de ChediakHigashi et la maladie de Gricelli.

\section{- Albinisme tyrosinase négatif}

Cet albinisme est caractérisé par un défaut de pigmentation cutanée oculaire entraînant une acuité visuelle très perturbée et le risque de cancer cutané par défaut de photoprotection. Le marqueur ultrastructural est l'absence de mélanosomes des stades
III et IV dans les mélanocytes du bulbe pilaire [20]. Une réaction à la Dopa en microscopie électronique peut être également pratiquée sur l'épiderme interfolliculaire [21].

\section{- Syndrome de Chediak-Higashi}

Cette affection est un albinisme partiel avec reflet métallique des cheveux. Il s'agit d'une anomalie généralisée des granules qui sont géants. La gravité de la maladie repose sur un déficit de l'immunité qui est létal avec décès par phase d'accélération dans la première décennie. Le diagnostic anténatal repose sur l'étude des mélanosomes qui, lors des diagnostics positifs, sont géants, à la fois dans le mélanocyte du bulbe pilaire et dans la tige pilaire. Une étude des granules des cellules des lignées blanche et plaquettaire peut également être faite. Le diagnostic le plus pratique de la maladie de ChediakHigashi actuellement est l'étude du cheveu en microscopie optique à la recherche des mélanosomes géants [22].

\section{Autres moyens de diagnostic anténatal pratique pour certaines maladies génétiques à expression cutanée}

Étude de la réparation de l'ADN après biopsie de trophoblaste

\section{- Xeroderma pigmentosum}

Le Xeroderma pigmentosum est une maladie autosomique récessive très fréquente dans les pays d'Afrique du Nord. Les enfants décèdent très tôt de multiples épithéliomas spinocellulaires invasifs et de mélanomes malins. Le diagnostic anténatal fut initialement pratiqué sur amniocytes cultivés et irradiés aux UV après amniocentèse à 16 semaines. Actuellement, ce diagnostic anténatal est assuré par Alain Sarasin (IGR, Villejuif, France) sur estimation du niveau de réparation de l'ADN sur biopsie de trophoblaste prélevé à 10 semaines de gestation [23].

\section{- Trichothiodystrophie avec photosensibilité}

Les patients atteints de cette grave génodermatose associant anomalies pilaires, retards staturo-pondéral et 


\section{RÉFÉRENCES}

18. Compton JG, DiGiovanna JJ, Santucci SK, Kearns KS, Amos CI, Abangan DL, Korge BP, McBride OW, Steinert PM, Balc SJ. Linkage of epidermolytic hyperkeratosis to the gene cluster on chromosome 12q. Nature Genet $1992 ; 1: 301-5$

19. Sarasin A, Blanchet-Bardon $\mathrm{Cl}$, Renault $G$, Lehmann A, Arlett $C$, Dumez Y. Prenatal diagnosis in a subset of trichothiodystrophy paticnts defective in DNA repair. Br J Dermatol $1992 ; 127$ : 485-91.

20. Eady R, Gunner D, Garner A, Rodeck C. Prenatal diagnosis of oculocutaneous albinism by clectron microscopy of fctal skin. J Invest Dermatol 1983 ; 80 : 210-2.

21. Shimizu H, Ishiko A, Kikuchi A, Akiyama M, Suzumori K, Nishikawa T. Prenatal diagnosis of tyrosinase-negative oculocutancous albinism. Lancet $1992 ; 340$ : 739-40.

22. Blanchet-Bardon $\mathrm{Cl}$, Durandy $\mathrm{A}$, Breton-Gorius J, Nazzaro V, Guigrand D Civatte J, Griscclli C. Diagnostic anténatal de la maladie de Chediak-Higashi. Ann Dermatol Venerol 1988 ; 115 : 258.

23. Sarasin A, Renault G, BlanchetBardon Cl, Boue J, Dumez Y. Le Xeroderma pigmentosum, caractéristiques cliniques, génétiques et cellulaires. Développement d'un test anténatal. médecine/sciences $1988 ; 4$. 608-17.

24. Sarasin A, Blanchet-Bardon Cl, Renault $G$, Lehmann A, Arlett C, Dumez Y. Prenatal diagnosis in a subset of trichothiodystrophy patients defective in DNA repair. Br J Dermatol 1992; 127 : 485-91.

25. Sybert VP, Holbrook KA, Levy M. Prenatal diagnosis of severe dermatologic mental, ont un taux de réparation de l'ADN réduit de façon similaire à ce qui est retrouvé dans le Xeroderma pigmentosum. Cette maladie peut donc également bénéficier d'un diagnostic anténatal par biopsie de trophoblaste à 10 semaines et étude de la réparation de l'ADN [24].

Dosage enzymatique sur biopsie de villosités choriales ou liquide amniotique [25]

Cette technique est općrationnelle dans certaines maladies génétiques à expression cutanée telles que la maladie de Fabry, les syndromes de Farber et de Hunter, la mucolipidose de type II, la maladie de Wolman.

Amniocentèse et culture des cellules amniotiques [25]

Ces deux examens sont un outil de diagnostic anténatal de certaines maladies génétiques à expression cutanée telles que le syndrome d'Ehler-Danlos de type VI, la fucosidose, la maladie de Gunther, la maladie de Refsum, l'anémie de Fanconi, le syndrome de Menkes.

\section{L'avenir}

L'avenir est déjà là, la liste des génodermatoses localisées sur la carte du génome humain s'allonge de semaine en semaine. Nous pensons que très rapidement, dans des génodermatoses graves comme certaines épidermolyses bulleuses et l'érythrodermie congénitale ichtyosiforme bulleuse, des diagnostics anténatals précoces à 10 semaines de gestation par biopsie de trophoblaste et génétique moléculaire seront possibles. La technique tardive par fotoscopie et microscopie électronique restera toujours opérationnelle pour les familles non informatives ou qui n'auront pu être étudiées

\section{Summary}

Prenatal diagnosis of genodermatoses

The rapid development of techniques available for fetal examination and tissue sampling, coupled with novel methods for enzymological, cytogenetic and DNA analysis has enabled the intrauterine detection of fetal skin disorders to become an integral part of the management of several genetic skin diseases. The relevance of new prenatal diagnostic methods to genetic diseases with major cutaneous manifestations is still largely limited to the detection of structural abnormalities in fetal skin biopsies (epidermolysis bullosa, keratinization disorders, pigmentation disorders). But the enormous advances in molecular biology of the epidermolysis bullosa group and the epidermolytic hyperkeratosis will soon allow identification of affected fetuses and possibly carriers.

\section{TIRÉS A PART}

Cl. Blanchet-Bardon. 